\title{
The Quality Monitoring of City Passenger Transportations on Regular Routes Using Data of Objective Control
}

\author{
Nikolay Trubin ${ }^{1}$, Nikolay Yakunin ${ }^{1}$, Natalia Yakunina ${ }^{1}$, and Igor Lyubimov ${ }^{1 *}$ \\ ${ }^{1}$ Orenburg State University, 13 Pobedy prosp., Orenburg, 460018, Russia
}

\begin{abstract}
The article presents the development justification of the quality monitoring methodology of city passenger transportations by motor transport on regular routes based on the criteria development for the evaluation of the passenger transportations quality with the use of the objective control means.
\end{abstract}

\section{Introduction}

Transport service of the population needs to be considered in continuous connection with the passengers' service quality. The quality coefficient of passenger transportations is the fullness coefficient of the rolling stock; costs of passengers' time during the movement; the traffic regularity; seriousness of the road accidents.

In this regard, the quality increase of passenger transportations by motor transport demands the implementation of the actions complex directed on the reduction of time cost for the population during the movement and the improvement of trips comfortableness.

The performed research is based on the researches analysis directed on the regularity study of the fullness of passenger motor vehicles (MV). The famous methods of the operative planning of passenger transport, directed on the optimization of route network, the improvement of the service quality are: the methods of natural survey of the rolling stock fullness which are carried out visually in different hours of days and days of the week [16, 17]; the calculating and tabular method consisting in the calculation of passengers' boarding and getting out on a stopping point and the registration in the draft table. This method is directed to the determination of passenger turnover of a definite stopping point, the movement regularity on stages [14-17]: the method of natural survey in the rolling stock being carried out by the researcher while travelling along the route who records the rolling stock fullness in the list of the stopping points. This method directed to the determination of the passenger traffic capacity on route stages and on hours of days [16, 17]: the method of the passengers inquiry. This method is connected with the passengers inquiry on a separate stopping point for the purpose of the determination of the transport connection with other stopping points $[16,17]$ : the method of the integrated survey of passenger traffic on the operating routes; the questionnaire method based on the completing of the specially draft

* Corresponding author: 1yubimovii@gmail.com 
questionnaires on the enterprises and in the institutions, residential districts. The main parameters of this method are the average range of the movement around the city, the correspondence between the city districts [4]; the reporting and statistical method based on the information about the proceeds from passenger transportation on routes and the sold tickets; the method of automatic calculation of passenger traffic.

\section{Material and methods}

The advantages of this method are: the assessment of passenger traffic density; the assessment of tendencies and forecasting; the assessment of the route change efficiency; the differentiation of the drivers' salary; the definition of "rush hours" and the formation of the optimum schedule for motor transport work.

The disadvantages of this method are: the high cost of the equipment; the need of personnel training for the system service; the measurement error of $7-10 \%$; the need of the passengers' accounting having privileges at journey.

Presently the existing methods, approaches and technical systems of monitoring for MV work on the route do not fully allow to realize the functions of MV management on the route taking into account the providing of the listed indicators.

In this regard, the subject of the scientific research directed to the technique development for the quality increase of city passenger traffic is urgent.

The model of the quality increase of passenger transportations by the motor transport on regular routes provides the admission system of the candidates to transport process (tenders) with the corresponding procedures which are implemented in many regions of the country and abroad and are directed to the tasks solution connected only with the tenders realization without mentioning the preparatory activities on the organization.

The algorithm of the organization of the candidates' admission to services rendering for the passenger transportations by motor transport on regular routes is developed on the basis of the system approach (figure 1).

The basic principles which are on the basis of the offered model are: a) primarily life, health, safety of citizens' property, ecological safety and secondary the economic results of the carrier; b) the availability to the population of the motor transport services on regular routes; c) guaranteeing on the territory of the constituent entity of the Russian Federation the unified transport policy on the base of the unified requirements and norms which are coordinated with the unified transport policy of the country; d) the equality of rights and obligations of legal persons and individual entrepreneurs at the implementation of the transport activity; e) the incentive of business and competition development; f) the providing of the quality standards for the passengers' service; g) the relief of the city route network; h) the use of the modern means of telematics; i) the compliance of the passengers' rights.

The algorithm provides three main logical stages for the organization of the candidates' admission to services rendering: the assessment of the information reliability provided by the candidate in the bid; the compliance assessment of the candidate's condition to the obligatory requirements which are in the legal framework of the federal and obligatory part of the regional level; actually tendering according to the motivating part of the regional legislation requirements consisting in the rolling stock assessment of the carrier and its organizational and functional structure $[16,17]$.

\section{Theory}

The first stage of the proposed algorithm realization is similar to the procedures in the existing system of the admission. 
The second stage is connected with the implementation of the obligatory requirements which are raised both to the vehicle and to the providing of the passengers safe transportations (figure 1).

The point system for the MV and the system of the safety providing assessment is applied on the third stage using scientifically based nomenclature of indicators and values $[12,13,17]$.

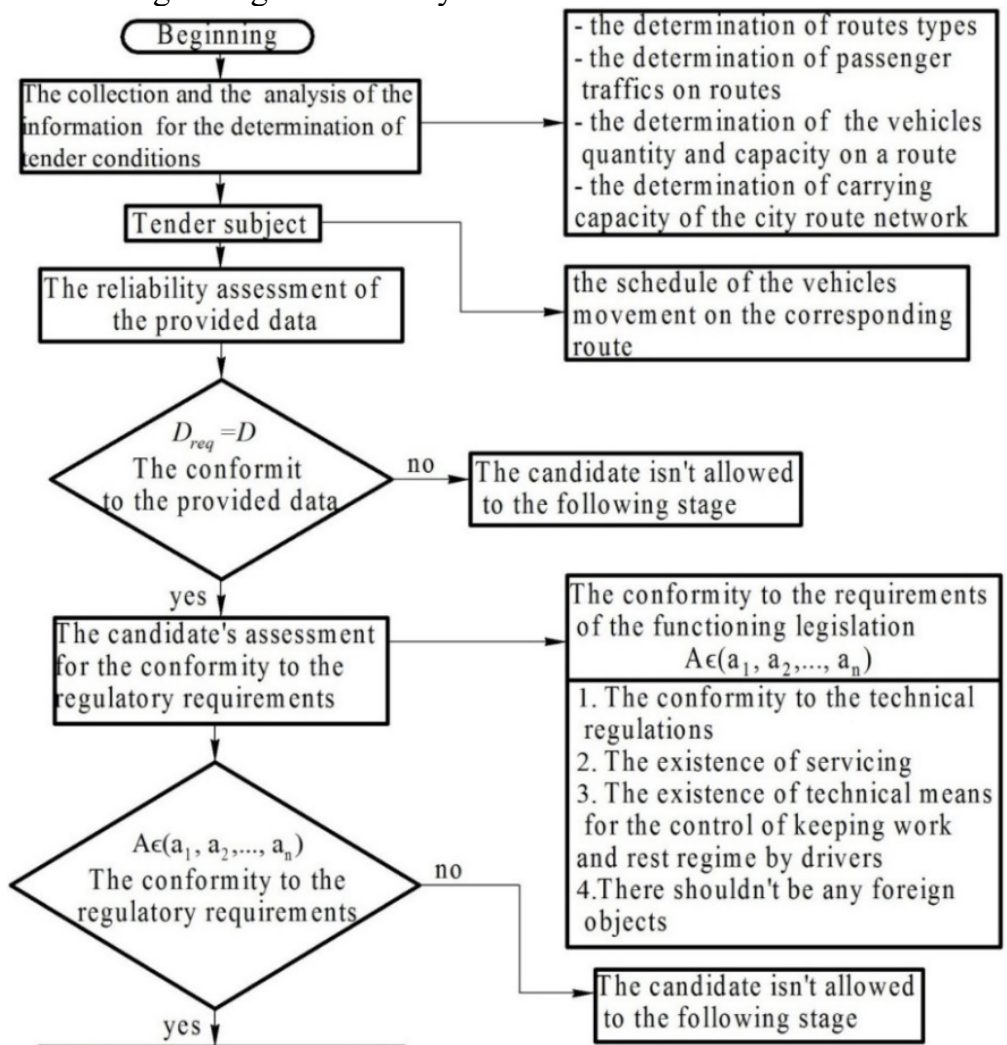

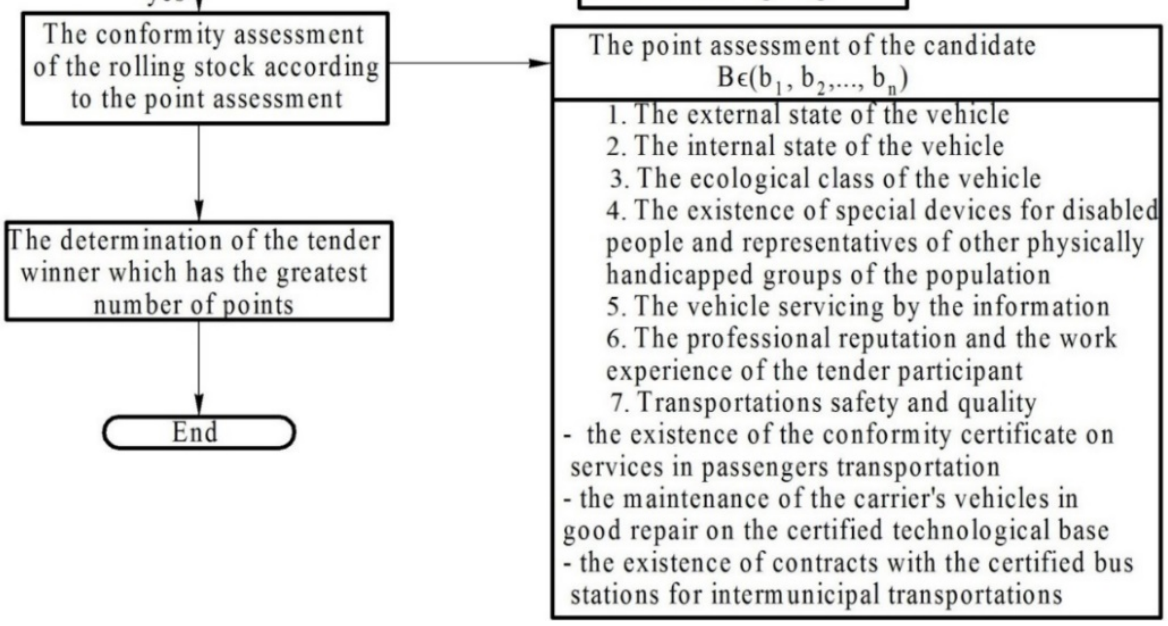

$D_{\text {req }}$ - the submitted data; $D$ - announced on a tender; $\mathrm{a}_{1 \ldots \mathrm{n}}$ - the regulatory requirements; $b_{1 \ldots n}-$ the requirements of the second stage

Fig 1. Algorithm of the organization of the applicants' admission to services rendering for passengers transportations by motor transport on regular routes. 
The indicators nomenclature for the point system was developed on the base of the method of experts' questionnaire in the field of the MV technical operation and the organization of the passengers transportations by motor transport who have the corresponding competence in the matters of the quality providing during transportations. Such choice is proved by the difference of other respondents' representation (for example, passengers) about the directions of the quality providing during transportations.

Two types of questionnaires were developed and used for the research: the influence of the technological support on the quality transportations; the influence of the rolling stock requirements on the quality transportations.

The questionnaires contain the experts' opinion according to the following indicators:

1) if the director has got motor transportation education and the experience of more than 5 years;

2) if the specialist who is responsible for the MV technical condition has got motor transportation education and the experience of more than 5 years;

3 ) if the specialist who is responsible for the transportations organization and safety has got motor transportation education and the experience of more than 5 years;

4) the average work experience for all bus drivers, the existence of various categories on vehicles driving;

5) the average work experience for bus drivers;

6) the objective monitoring results of the observance of the operational indicators for MV work (speed, regularity, schedule and routes);

7) the conducting of the road accidents accounting because of drivers as the tender candidate;

8) the existence of the conformity certificate on services in passengers transportation by motor transport;

9) the maintenance of the carrier's vehicles in good repair on the certified technological base;

10) the existence of contracts with the certified bus stations at the implementation of intermunicipal transportations;

11) the use of transport electronic cards;

12)the average point assessment of the rolling stock according to the inspection certificates.

The MV technical condition was evaluated by the following indicators:

1) the MV external state (existence of corrosion and mechanical damages, condition of paint and varnish covering);

2) the MV equipment by special devices for disabled people and representatives of other physically handicapped groups of the population;

3) the MV internal state (good fixing of hand-rail and handles, illumination, integrity of seats covering, the state of floor covering, the sanitary condition);

4) the MV ecological class;

5) the existence and operability of climatic installation.

The sixteen-point scale was used for the quantitative assessment of the proposed indicators.

Sixteen points were given to the indicator that is maximum influence the transportation quality, zero point - for the indicator with the absence of the influence the transport process quality.

We previously used the prompts matrix for the distortions reduction of the basic data. The criterion of mistaken elements removal was the existence of several zero in an element line. The elements of the prompt matrix were determined by a formula: 


$$
k_{j}=\sum_{i=1}^{n} b_{i j}, j=\overline{1, n},
$$

where $b_{i j}$ - the cell value of the questionnaire. value:

The prompt matrix is a line in which the indicator with the greatest value is equal to the

$$
k_{\max }=\max _{j} k_{j},
$$

and all elements are transformed by the following formula:

$$
v_{i j}=\frac{b_{i j} k_{\max }}{k_{j}}, i=\overline{1, m, j}=\overline{1, n} .
$$

The fuzzy multiplicity $A_{j}$ was set for each indicator corresponding to the opinions totality of respondents on the importance of $\mathrm{j}$-indicator.

Taking into account the frequency of $v_{i j}$ occurance of the $i$-assessment for $j$-indicator we define the fuzzy multiplicity:

$$
A_{j}=\left.\left\{i \mid \mu_{j}(i)\right\}\right|_{i=0, n}
$$

where $\mu_{j}(i)$ - the membership function, which is determined by a formula:

$$
\mu_{j}(i)=\frac{v_{i j}}{\sum_{k=1}^{m} v_{i k}}, j=\overline{1, m},
$$

where $n$ - the maximum point according to the respondents' assessment.

We determine the parameter with the largest influence $\alpha_{j}$ where the mathematical foresight of point is the greatest:

$$
\mathrm{M}_{\mathrm{j}}=\sum_{i \in \operatorname{supp} A_{j}} i \cdot \mu_{j}(i)
$$

where $\mu_{j}(i)$ - the membership function of $A_{j}$, supp $A_{j}-$ a fillet of the fuzzy multiplicity of $A_{j}$.

Similarly we calculate the other mathematical foresights. We make the comparison of the fuzzy multiplicity by the similarity index:

$$
s_{j}=S\left(A_{j}, A_{z}\right)=\frac{\left|A_{j} \cap A_{z}\right|}{\left|A_{j} \cup A_{z}\right|},
$$

where $\mathrm{s}_{\mathrm{j}}$ - similarity measure of the fuzzy multiplicity of $A_{j}$ and $A_{j},(j=\overline{1, m}, \quad j \neq$ 12); $z$ - a serial number of the member with the maximum mathematical foresight.

Capacity of the fuzzy multiplicity of $A$ ( $A$ is either $A_{j} \cap A_{z}$ or $A_{j} \cup A_{1}$ ):

$$
\begin{gathered}
|A|=\sum_{i \in \operatorname{supp} \hat{A}} \mu_{A}(i), \\
A_{j} \cap A_{z}=\left\{\left(i \mid \min \left(\mu_{A_{j}}(i), \mu_{A_{z}}(i)\right)\right)\right\} \\
A_{j} \cup A_{z}=\left\{\left(i \mid \max \left(\mu_{A_{j}}(i), \mu_{A_{z}}(i)\right)\right)\right\}
\end{gathered},
$$

The influence of importance we determine by a formula: 


$$
\alpha_{j}=\frac{s_{j}}{\sum_{k=1}^{m} s_{k}} .
$$

Taking into account the found values of the influenced coefficients $\alpha_{j}$ for $j=\overline{1, m}$ we define the average point of the vehicle $(P v)$ by a formula:

$$
P v=\frac{\sum_{n=1} \sum_{j=1} \alpha_{j}^{\prime}}{n},
$$

where $\alpha_{j}{ }^{\prime}$ - the charged points at the assessment of the vehicle indicators; $n$ - the number of vehicles which a carrier has, unit.

The summable indicators of the point assessment of the candidate $(P)$ are determined by a formula:

$$
P=P v+\sum_{j=1}^{11} \alpha_{j},
$$

where $\alpha_{j}$ - the charged points at the indicators assessment of the technological readiness of the candidate on passenger transportations.

Except the indicators with the positive influence, the indicators negatively influencing the passenger transportations quality were also researched. They are:

- the number of the road accidents $(N)$ because of the carrier's driver with the serious consequences or caused the extensive material damage (during 12 months preceding a tender);

- the number of traffic abuses by the drivers $(D)$ on carrier's vehicles $(V)$ according to the State Inspection for Road Traffic Safety $(z)$.

Taking into account the requirements of the antitrust law, the guarantee of the approach objectivity we consider not only the absolute indicators, but also the relative indicators to one vehicle $[7,8,9,10,11]$.

The relative indicator $m_{R A}$ showing the number of road accidents is connected with the absolute indicator $N$

$$
m_{R A}=\frac{N}{V C_{\mathrm{RA}}}
$$

where $C_{R A}$ - the correcting coefficient depending on the road accidents number which have happened because of a carrier.

The absolute indicator "the abuses number of traffic regulations by the drivers $(D)$ on the carrier's rolling stock according to the State Inspection for Road Traffic Safety" is transferred to the relative indicator of $z_{\operatorname{TrR}}$ :

$$
z_{T r R}=\frac{D}{V C_{T r R}}
$$

where $C_{T r R}$ - the correcting coefficient characterizing the abuses of traffic regulations by carrier's drivers.

The values of the correcting coefficients $C_{R A}$ and $C_{T r R}$ are found on carrier(s) with the best indicators of traffic safety (the mathematical foresight of indicators: "the number of the road accidents on one unit of the rolling stock"; "the number of traffic abuses on one unit of the rolling stock" are less than 0,1 ), having all necessary services on traffic safety. 


$$
\left\{\begin{array}{l}
\frac{N}{V} \Rightarrow \min \\
\frac{D}{V} \Rightarrow \min
\end{array}\right.
$$

The values calculation of the correcting coefficients $C_{R A}$ and $C_{T r R}$ of the best economic enterprise (with an exception of the indicators influence $m$ and $z$ on the general total point assessment) is represented as justified because it excludes the influence of distinctions between the large and small carriers.

The application of $m$ and $z$ indicators is necessary to increase the transport process safety and they motivate a carrier to observe traffic regulations, to decrease the number of the road accidents $[1,2,3,5-8]$.

The experimental studies about the carriers were carried out in 2009 and in 2013 according to the requirements of the tendering documentation.

The total amount of points was determined by the indicators of the tendering documentation of carriers in 2009 and 2013 [17].

Basing on the indicators given in the table the indicators changes were defined while characterizing the carriers at tendering in 2009 and 2013.

The indicators changes are presented in table 1.

Table 1. The indicators changes being estimated at tendering characterizing the carriers in 2009 and 2013

\begin{tabular}{|c|c|c|c|}
\hline \multirow{2}{*}{ Indicator number } & \multicolumn{2}{|c|}{ Indicators values in } & \multirow{2}{*}{ Changes of indicators values, \% } \\
\cline { 2 - 4 } & $\mathbf{2 0 0 9}$ & $\mathbf{2 0 1 3}$ & 39,8 \\
\hline 1 & 1,58 & 2.21 & 1,97 \\
\hline 2 & 1,52 & 1,55 & $-8,7$ \\
\hline 3 & 0,69 & 0,63 & $-12,2$ \\
\hline 4 & 0,74 & 0,65 & 16,87 \\
\hline 5 & 0,83 & 0,97 & 45,16 \\
\hline 6 & 0,62 & 0,90 & 50 \\
\hline 7 & 1,38 & 2,07 & 393 \\
\hline 8 & 0,28 & 1,38 & 4,61 \\
\hline 9 & 1,52 & 1,59 & 7,83 \\
\hline 10 & 1,66 & 1,79 & 27,19 \\
\hline 11 & 1,14 & 1,45 & 16,03 \\
\hline 12 & 1,31 & 1,52 & 0 \\
\hline 13 & 0 & 0 & 34,5 \\
\hline 14 & 6,39 & 8,59 & $-9,5$ \\
\hline Average quantity of vehicles & 60,93 & 55,14 & 33,5 \\
\hline at one carrier, unit & 17,2 & 22,72 & \\
\hline Total amount of points & & & \\
\hline
\end{tabular}

\section{Results and Discussion}

The analysis of the received results confirms the increase in indicators of carriers' tendering of passenger transportations by motor transport. 


\section{References}

1. Aboudolas, K., Papageorgiou, M., Kosmatopoulas, E.B. Store-and-forward based methods for the signal control problem in large scale urban road networks. Transportation Research Part C, 17 (2), 163 - 174 (2009)

2. Yakunin N.N., Yakunina N.V., Spirin A.V. Analysis of the federal law provisions of the Russian Federation from 13.07.2015 № 220 from the position of the methodology about the improvement of the passenger transportations quality by motor transport. Intellect. Innovations. Investments. № 2. Pp. 128-132 (2016)

3. Bondarenko, E.V., Goncharov, A.A., Gorlatov, S.E. Lyubimov, II, Manaev, K.I., Melnikov, A.N., Trubin, N.A. Concept of municipal road transport. The world of transport and technological machines, 2 (49). Orel, 110-116 (2015)

4. Le, T., Kovacs, P., Walton, N., Vu, H. L., Andrew, L. H., Hoogendoorn, S. P., Decentralised Signal Control for Urban Road Networks. arXiv: 1310.0491v2 [math.OC] (2014).

5. Liu, R. Traffic Simulation with DRACULA. In Fundamentals of Traffic Simulation (Ed. J Barcelo), Chapter 8. Springer. ISBN: 978-1-4419-6141-9. Pp 295-322 (2010)

6. Liu, R., Smith, M. J. Route choice and signal control: a study of the stability and instability of traffic signal controlled networks. Transportation Research Part B, 77, 123 145 (2015)

7. Liu, R., van Vliet, D. and Watling, D. Microsimulation models incorporating both demand and supply dynamics. Transportation Research, 40A, 125-150 (2006)

8. Lyubimov, I.I. Indicators demand for urban passenger transport (by the example of Orenburg). Bulletin of the Orenburg State University, 9 (115), 139-143 (2009)

9. Lyubimov, I.I., Sultanov, N.Z., Bondarenko ,Ye.V. To the subject of the rational structure formation of the city passenger transport in Orenburg. Vestnik of the Moscow automobile and road construction state technical university (MADI). № 3. Pp. 21-25 (2009).

10. Lyubimov, I.I., Sultanov, N.Z. Theoretical bases of perfection transport system. Bulletin of the Orenburg State University, 10, Pp. 121-123 (2014)

11. Lyubimov, I.I., Melnikov, A.N., Trubin, N.A. The Control System Improvement of the City Motor Transportation. Science Direct: Procedia Engineering. Volume 150. - Pp. 1192-1199 (2016).

12. Melnikov, A.N., Lyubimov, I.I., Manayev, K.I. Improvement of the Vehicles Fleet Structure of a Specialized Motor Transport Enterprise. Science Direct: Procedia Engineering. Volume 150. Pp. 1200-1208 (2016)

13. Varaiya, P. 2013. Max pressure control of a network of signalised intersections. Transportation Research Part C, 177-195 (2013)

14. Yakunin N.N., Yakunina N.V., Kuysokov T.A. Theoretical research of an indicator " $a$ dynamic dimension of a passenger” Intellect. Innovations. Investments. № 1. Pp. 95-97 (2016)

15. Yakunina, N.V. Passenger transportations by motor transport on regular routes: Theoretical fundamentals of the methodology for the quality improvement. Standards and quality. № 2. Pp. 92-93 (2015)

16. Yakunina, N.V. Methodology of the improvement quality of passenger transportation by motor transport on regular routes: Monograph. Orenburg: Publ. company «University». 262 p. (2015) 
17. Yakunin, N.N., Yakunina, N.V., Shakhalevich, G.A. Certification on the motor transport: textbook. - Orenburg: OSU. 583 p. (2015) 